\title{
Determinación de fenilalanina y galactosa total a partir de una muestra de sangre seca en papel de filtro: aplicación al tamizaje neonatal
}

\author{
Eneida Torres, Arlenys Baloy, Amarilys Frómeta, Lianny Fernández \\ Laboratorio de Pesquisaje Neonatal, Centro de Inmunoensayo, Ciudad de La Habana, Cuba.
}

Los programas de tamizaje neonatal para alteraciones metabólicas deben incluir como mínimo la detección de fenilcetonuria e hipotiroidismo congénito. En este estudio evaluamos un método que cuantifica el valor de galactosa total (Gal) y fenilalanina (Phe) a partir de un disco de sangre seca en papel de filtro, eluido con agua destilada, previa desproteinización con metanol-acetona; posteriormente, se transfirieron $10 \mathrm{uL}$ del eluido a una placa ultramicroELISA y se le añadió la mezcla de reactivos para determinar Phe; al remanente se le añadieron los reactivos para cuantificar Gal. El método fue lineal en un rango de concentración de 0-50 mg/dL para Phe y 0$60 \mathrm{mg} / \mathrm{dl}$ para Gal; el límite de detección para Phe/Gal fue de 0,14/0,9 mg/dl; se evaluaron tres muestras con niveles bajo, medio y elevado de Phe/Gal y se obtuvo una imprecisión intraensayo de $6,8 \pm 1,7 / 7,8 \pm 2 \%$ y una imprecisión interensayo de $5,4 \pm 0,7 / 7,5 \pm 1,8 \%$, respectivamente. La recuperación analítica fue de 100,2\% $\pm 1,9 \%$ para Gal y $100,3 \% \pm 1,7 \%$ para Phe. No se evidenciaron interferencias con los antibióticos evaluados. Se realizó un estudio con 1.000 muestras de neonatos entre las que se encontraron cuatro muestras con concentraciones de Phe y dos con concentraciones de Gal por encima de los niveles de corte para estos analitos. Se obtuvo una excelente correlación lineal entre los dos métodos con que fueron evaluadas de forma comparativa las muestras (UMTEST PKU e ICN GAL-M W EA). Este método permite incorporar una enfermedad metabólica de baja incidencia (galactosemia 1:30.000), en un programa de tamizaje masivo para la detección de fenilcetonuria, lo que justificaría el costo del tamizaje neonatal.

Palabras clave: galactosemia, fenilcetonuria, tamizaje neonatal.

Phenylalanine and total galactose measurement from dried blood spotted on filter paper application to newborn screening

Neonatal screening programs for metabolic disorders are recommended especially for phenylketonuria and congenital hypothyroidism. The present study was designed to adapt, develop and evaluate a SUMA method for total galactose (Gal) and phenilalanine (Phe) measurement on filter paper blood specimens. A single $5 \mathrm{~mm}$ disk with blood was deproteinized with methanolacetone and eluted with distilled water. Ten $\mu$ of the extract was transferred to one well of a ultramicroELISA plate, and the reaction solution was added to determine Phe level. The remaining extract was used for the GAL determinations. The method showed good linearity in a 0-50 mg/dl concentration range for Phe and 0-60 mg/dl for Gal. The detection limit was $0.14 \mathrm{mg} / \mathrm{dl}$ for Phe and $0.9 \mathrm{mg} / \mathrm{dl}$ for Gal. Reproducibility was assessed with filter paper blood specimens containing Gal and Phe at low, middle and high levels. Intraassay coefficients of variation were $10 \%, 7.5 \%$, $6.22 \%$, and $8.5 \%, 7 \%, 5 \%$, respectively, whereas interassay coefficients of variation were $9.54 \%$, $6 \%, 7 \%$ and $6 \%, 4.6 \%, 5,6 \%$, respectively. In 1,000 samples from newborns, four samples of Phe and two samples of Gal showed a concentration below the treshold set for each assay. This method provides a rapid means to survey for a low incidence disease (i.e., galactosaemia: incidence, 1/30,000), in existing phenylketonuria analysis programs, where an incidence of 1 / $10,000)$, easily justifies the cost of mass screening.

Key words: galactosaemia, phenylketonuria, neonatal screening. 
Un programa de tamizaje neonatal para alteraciones metabólicas debe incluir como mínimo la detección de fenilcetonuria e hipotiroidismo congénito. Estas alteraciones producen retardo mental severo y una morbilidad importante que incluso lleva a la muerte; además, una vez constituido, es importante incluir otras afecciones metabólicas como la galactosemia, la enfermedad jarabe de arce, el déficit de biotinidasa y otras cuyo diagnóstico precoz, a pesar de su baja frecuencia en la población, puede cambiar el curso del proceso patológico y evitar la mayoría de las complicaciones, incluido el retardo mental (1).

De ahí, la necesidad de incrementar la sensibilidad de los ensayos diseñados para los tamizajes masivos de enfermedades congénitas, sin descuidar el ahorro económico que cobra importancia en estos tiempos.

Estudios recientes han demostrado la factibilidad de las pruebas de marcación doble, como los ensayos multianalito que permiten estudiar varios analitos simultáneamente en el mismo procedimiento y otras como la técnica descrita por Hoffman y col. en la cual se comparte el eluido obtenido de una misma muestra para medir simultáneamente las concentraciones de galactosa total (Gal) y L-fenilalanina (Phe) (2).

En este estudio nos propusimos desarrollar, evaluar y adaptar a la tecnología SUMA, dos ensayos simples que comparten el eluido obtenido de una misma muestra de sangre seca en papel de filtro, para analizar posteriormente los valores de Gal y Phe, método que se puede usar en los programas masivos de tamizaje neonatal para galactosemia y fenilcetonuria.

\section{Materiales y métodos}

\section{Preparación combinada de los calibradores y controles de Phe y Gal}

Los calibradores y controles empleados se prepararon a partir de sangre total humana procedente de donantes voluntarios, negativa a

\section{Correspondencia: \\ Eneida Torres \\ Calle 134 y Avenida 25; apartado aéreo 6653 \\ Ciudad de La Habana, Cuba}

Recibido: 17/07/01; aceptado: 15/02//02 las pruebas de detección de anti-VIH 1 y $2, \mathrm{HbsAg}$ y HCV y ajustada a un valor de hematocrito de $55 \%$ con el objetivo de simular el hematocrito neonatal. Se adicionaron cantidades necesarias de Gal y Phe y se obtuvieron calibradores con concentraciones de 5/2,97, 10/5,95, 20/11,89, $30 / 23,79$ y $60 / 47,5 \mathrm{mg} / \mathrm{dl}$, respectivamente. El control del ensayo tenía una concentración de 8 $\mathrm{mg} / \mathrm{dl}$ para Gal y $15,5 \mathrm{mg} / \mathrm{dl}$ para Phe.

Al mismo tiempo, se prepararon controles de concentraciones bajas $(2 / 9 \mathrm{mg} / \mathrm{dl})$, medias $(15 / 18$ $\mathrm{mg} / \mathrm{dl})$ y altas $(40 / 30 \mathrm{mg} / \mathrm{dl})$ de Gal/Phe, respectivamente.

\section{Preparación de controles Gal/Phe/antibióticos para evaluar la especificidad del método}

Se prepararon 14 controles con sangre total humana ajustada a un valor de hematocrito de $55 \%$; a cada uno se le añadió una cantidad basal de Gal/Phe de $8 / 3 \mathrm{mg} / \mathrm{dl}$ y una concentración de $100 \mathrm{mg} / \mathrm{dl}$ del antibiótico por evaluar: penicilina cristalina, penicilina benzatínica, penicilina procaínica, ampicilina, eritromicina, ofloxacina, cloranfenicol, gentamicina, kanamicina, azitromicina, sulfametoxazol, amoxicilina, oxacilina y cefalexina; además, se preparó un control normal de Gal/Phe (8/3 mg/dl).

Todas las muestras se dispensaron en tarjetas de papel de filtro Schleicher Schuell 903 y se colocaron en posición horizontal sobre un soporte diseñado con el propósito de evitar el contacto con la superficie; se dejaron secar durante 24 horas a temperatura ambiente $\left(20^{\circ} \mathrm{C}-30{ }^{\circ} \mathrm{C}\right)$ y con control de la humedad relativa $\left(<70{ }^{\circ} \mathrm{C}\right)$; posteriormente, se guardaron en sobres de papel y se conservaron a - $20^{\circ} \mathrm{C}$ hasta su análisis.

\section{Muestras}

En el método combinado, se evaluaron 1.000 muestras de sangre seca sobre papel de filtro de recién nacidos procedentes del Programa Nacional de Pesquisaje Masivo de Fenilcetonuria.

\section{Estudio de estabilidad}

Para este estudio, los calibradores combinados de Gal/Phe en sangre seca sobre papel de filtro se almacenaron en cuatro condiciones diferentes: 
1) $(\mathrm{C} 1)=-20^{\circ} \mathrm{C}$; 2) $(\mathrm{C} 2)=4^{\circ} \mathrm{C} ; 3(\mathrm{C} 3)=37^{\circ} \mathrm{C}, \mathrm{y} \quad$ Método

4) $(\mathrm{C} 4)=$ temperatura ambiente $\left(20\right.$ a $\left.30^{\circ} \mathrm{C}\right)$.

\section{Equipos y accesorios}

Se emplearon los siguientes equipos y accesorios de la tecnología SUMA, fabricados en el Centro de Inmunoensayo, La Habana, Cuba:

- P-51: perforador de discos de papel de $5 \mathrm{~mm}$ de diámetro.

- Lector de placas fluorímetro-fotómetro PR-521 (rango espectral de fluorescencia: 410-500 nm).

- Strips reader software, versión, 7.0, soportado sobre Windows.
Para este procedimiento (figura1) se usaron discos de sangre seca en papel de filtro con un diámetro de $5 \mathrm{~mm}$; los analitos de interés (Gal y Phe) fueron eluidos del papel con $40 \mu \mathrm{l}$ de agua destilada, durante 1 hora con agitación y previa fijación de la hemoglobina al disco con una mezcla $\mathrm{v} / \mathrm{v}$ de metanol-acetona durante 25 minutos a $37^{\circ} \mathrm{C}$; después de eluidos los analitos de una muestra común, se transfirieron $10 \mu \mathrm{l}$ del eluido a uno de los pocillos de una placa ultramicroELISA y se le añadió la mezcla de reactivos para determinar Phe

$15 \mu \mathrm{l}$ metanol/acetona $\mathrm{v} / \mathrm{v}$

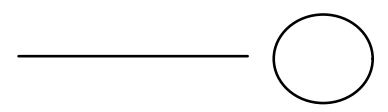

Disco de $5 \mathrm{~mm}$

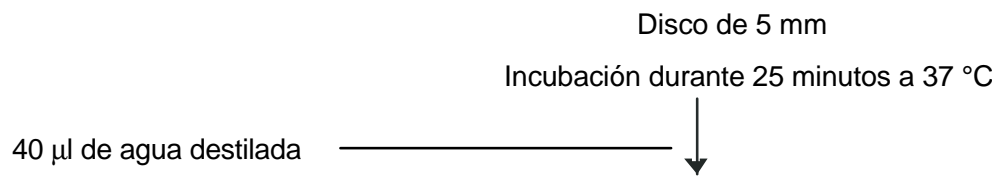

Agitación durante 1 hora a temperatura ambiente

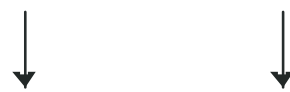

$10 \mu \mathrm{l}$ de eluido Reactivos para determinar Gal

Reactivos para determinar Phe
Tampón succinato
Ninhidrina
L-leucil-L-alanina

Incubación durante 1 hora a $62^{\circ} \mathrm{C}$

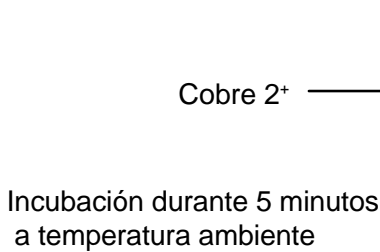

Incubación durante 5 minutos

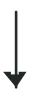

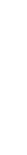
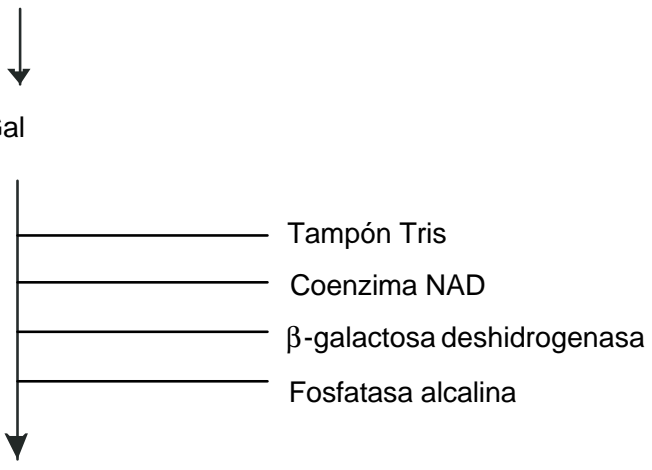

Incubación durante 1 hora a $37^{\circ} \mathrm{C}$

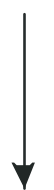

Lectura en el fluorímetro-fotómetro PR 521

Lectura en el fluorímetro-fotómetro PR 521

Figura 1. Diagrama para el procedimiento del método. 
con base en la reacción de la ninhidrina (3); al remanente se le añadieron los reactivos para determinar Gal acoplada a las reacciones catalizadas por la fosfatasa alcalina (EC 3.1.3.1) y la b-galactosa deshidrogenasa (EC 1.1.1.48) (4).

\section{Determinación de Phe}

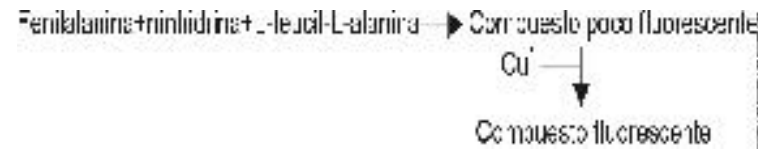

En cada uno de los pocillos de la placa ultramicroELISA, los $10 \mathrm{ml}$ de eluido que fueron transferidos reaccionaron con $10 \mathrm{ml}$ de la mezcla de reactivos compuesta por ninhidrina, tampón succinato y el dipéptido L-leucil-L-alanina, formando un complejo poco fluorescente en condiciones óptimas de $\mathrm{pH}$ y temperatura. La adición de $10 \mathrm{ml}$ del reactivo de cobre produce la amplificación de la fluorescencia. La intensidad de la fluorescencia es directamente proporcional a la concentración de Phe presente en la muestra (3).

\section{Determinación de Gal}

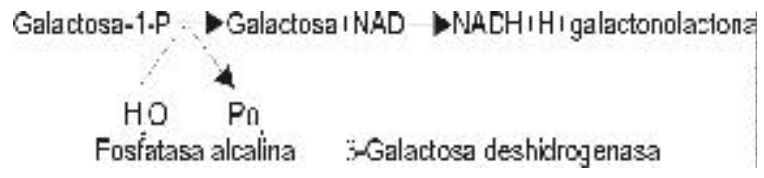

Después de transferir $10 \mathrm{ml}$ del eluido a un pocillo de la placa ultramicroELISA para determinar Phe, al eluido remanente se le añaden $30 \mathrm{ml}$ de una mezcla compuesta por la coenzima nicotinamida adenina dinucleótido (NAD), las enzimas bgalactosa deshidrogenasa (EC 1.1.1.48) y fosfatasa alcalina (EC 3.1.3.1) y una solución tampón; la galactosa-1-fosfato es hidrolizada por la fosfatasa alcalina a galactosa libre, la cual es oxidada por la enzima b-galactosa deshidrogenasa, que genera durante su actividad al NAD reducido $(\mathrm{NADH}+\mathrm{H})$, reacciones que ocurren en condiciones óptimas de $\mathrm{pH}$ y temperatura. La intensidad de la fluorescencia emitida por el $\mathrm{NADH}+\mathrm{H}$ es directamente proporcional a la concentración de Gal presente en la muestra (4).

Se realizó la medición de la fluorescencia en un lector fluorímetro-fotómetro PR-521 y los resultados se calcularon automáticamente empleando un programa diseñado para el cálculo de la concentración e interpretación de los resultados, soportado sobre Windows.

\section{Resultados}

El método fue lineal en un rango de concentración de $0-50 \mathrm{mg} / \mathrm{dl}$ para Phe y $0-60 \mathrm{mg} / \mathrm{dl}$ para Gal (figura 2), lo cual permitió la detección de estos analitos en la muestra, siempre que se encontraran dentro de estos niveles de concentración.

La precisión del método se calculó evaluando durante 10 días 20 réplicas de controles comprendidos en tres niveles de concentraciones de Gal/Phe: 2/9, 15/18 y 40/30 mg/dl, con un coeficiente de variación (CV) intraensayo de 10/ $8,5,7,5 / 7$ y $6,22 / 5$ y un CV interensayo $9,54 / 6,6 /$ 4,6 y $7 / 5,6$, respectivamente (cuadro 1).

El porcentaje de recuperación obtenido al añadir diferentes cantidades de Gal/Phe a tres muestras de sangre, posteriormente dispensadas en papel de filtro fue de 100,2 / 100,3\%, respectivamente (cuadro 1).

El límite de detección del método para Phe fue de $0,14 \mathrm{mg} / \mathrm{dl}$ y para Gal de $0,9 \mathrm{mg} / \mathrm{dl}$. Se definió como la concentración calculada para la fluorescencia equivalente al calibrador de concentración $0 \mathrm{mg} / \mathrm{dl}$ de Gal/Phe más dos desviaciones estándar (DE).

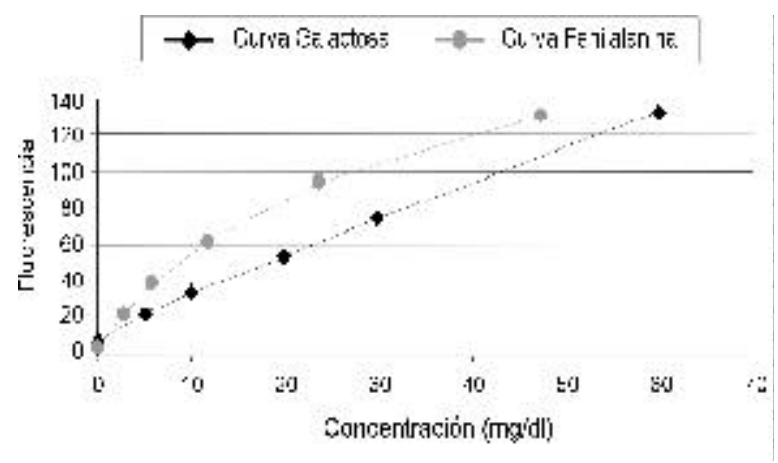

Figura 2. Curva de calibración para galactosa total y fenilalanina.

Ecuación de la recta obtenida para la curva de galactosa $(y=2,0366 x+11,768)$

Ecuación de la recta obtenida para la curva de fenilalanina $(y=2,5354 x+20,081)$ 
Cuadro 1. Evaluación de la precisión y la recuperación analítica del método combinado para determinar galactosa total y fenilalanina.

\begin{tabular}{|c|c|c|c|c|c|c|c|c|}
\hline \multirow{3}{*}{$\begin{array}{l}\text { Gal/Phe } \\
\text { (mg/dl) }\end{array}$} & \multicolumn{4}{|c|}{ Precisión } & \multicolumn{4}{|c|}{ Estudio de recuperación } \\
\hline & \multicolumn{2}{|c|}{ Intraensayo } & \multicolumn{2}{|c|}{ Interensayo } & \multirow{2}{*}{$\begin{array}{l}\text { Muestras } \\
\text { Gal/Phe }\end{array}$} & \multicolumn{2}{|c|}{$\begin{array}{cc}\text { Concentración }(\mathrm{mg} / \mathrm{dl}) \\
\text { Esperada }\end{array}$} & \multirow[t]{2}{*}{$\mathbf{R}(\%)$} \\
\hline & CV (\%) & DE & CV (\%) & DE & & & & \\
\hline $\begin{array}{l}2 / 9 \\
15 / 18\end{array}$ & $\begin{array}{c}10 / 8,5 \\
7,5 / 7\end{array}$ & $\begin{array}{c}0,7 / 0,5 \\
0,96 / 0,79\end{array}$ & $\begin{array}{l}9,54 / 6 \\
6 / 4,6\end{array}$ & $\begin{array}{l}0,74 / 0,4 \\
0,78 / 0,7\end{array}$ & $\begin{array}{l}1 \\
2\end{array}$ & $\begin{array}{c}2 / 9 \\
15 / 18\end{array}$ & $\begin{array}{c}2,2 / 8,82 \\
14,46 / 18,5\end{array}$ & $\begin{array}{c}110 / 98 \\
97,3 / 102,8\end{array}$ \\
\hline $40 / 30$ & $6,22 / 5$ & $1,20 / 1,15$ & $7 / 5,6$ & $1,46 / 1,4$ & 3 & $40 / 30$ & $37,4 / 30,05$ & $93,5 / 100,1$ \\
\hline
\end{tabular}

CV: coeficiente de variación; DE: desviación estándar; R: recuperación

Cuadro 2. Evaluación de antibióticos en el método combinado para determinar galactosa total y fenilalanina.

\begin{tabular}{lccc}
\hline Antibióticos & $\begin{array}{c}\text { Cantidad de antibióticos } \\
\text { añadidos }(\mathbf{m g} / \mathbf{d l})\end{array}$ & $\begin{array}{c}\text { Cantidad basal de } \\
\text { Gal/Phe añadida } \\
\text { (mg/d) }\end{array}$ & $\begin{array}{c}\text { Resultados obtenidos en el } \\
\text { método combinado } \\
\text { (mg/d) }\end{array}$ \\
\hline Penicilina cristalina & 100 & $8 / 3$ & $7,3 / 3$ \\
Penicilina procaínica & 100 & $8 / 3$ & $7,2 / 3$ \\
Penicilina benzatinica & 100 & $8 / 3$ & $8 / 2,8$ \\
Cefalexina & 100 & $8 / 3$ & $7,1 / 2,9$ \\
Ampicilina & 100 & $8 / 3$ & $7,5 / 2,8$ \\
Amoxicilina & 100 & $8 / 3$ & $8 / 3,1$ \\
Oxacilina & 100 & $8 / 3$ & $8 / 2,9$ \\
Sulfametoxazol & 100 & $8 / 3$ & $7,4 / 2,8$ \\
Eritromicina & 100 & $8 / 3$ & $8,1 / 2,9$ \\
Ofloxacina & 100 & $8 / 3$ & $7,9 / 3$ \\
Gentamicina & 100 & $8 / 3$ & $7,2 / 3$ \\
Kanamicina & 100 & $8 / 3$ & $7,9 / 2,8$ \\
Cloranfenicol & 100 & $8 / 3$ & $7,5 / 2,7$ \\
Azitromicina & 100 & $8 / 3$ & $8 / 2,9$ \\
\hline
\end{tabular}

Se evaluó la posible interferencia que podían ejercer en nuestro método 14 antibióticos usados con frecuencia en la etapa neonatal a concentraciones que exceden las dosis máximas terapéuticas en sangre, en las condiciones normales del método empleado. En el cuadro 2 se muestran los resultados obtenidos.

En el estudio de población realizado con 1.000 muestras de sangre seca sobre papel de filtro recolectadas en recién nacidos, se obtuvo una media para Phe de $0,8 \mathrm{mg} / \mathrm{dl}$ y $4,8 \mathrm{mg} / \mathrm{dl}$ para Gal y una DE de 0,6 para Phe y 1,7 para Gal (figuras 3 y 4); 4 de las muestras analizadas mostraron una concentración de Phe por encima del nivel de corte obtenido para este analito ( $3 \mathrm{mg} / \mathrm{dl})$, las cuales se evaluaron de forma comparativa en el ensayo simple UMTEST PKU (5), dando una ecuación de regresión y=0,9077x $+0,6804$ r2 = 0,9853 , donde y son los resultados de concentración $(\mathrm{mg} / \mathrm{dl})$ de Phe en el método combinado; $x$, los resultados de concentración $(\mathrm{mg} / \mathrm{dl})$ de Phe en el UMTEST PKU, con una buena correlación lineal $(r=0,998)$ (figura 5); además, dos muestras tuvieron una concentración de Gal por encima del nivel de corte diagnóstico obtenido en nuestro método para Gal (10 mg/dl); los resultados obtenidos fueron corroborados evaluándolos con el método colorimétrico comercial ICN GAL-M W EA; se obtuvieron resultados que evidencian una buena correlación entre ambas técnicas (13,9 mg/dl para una de las muestras y $14,2 \mathrm{mg} / \mathrm{dl}$ para la otra, con el estuche comercial; $13 \mathrm{mg} / \mathrm{dl}$ y $14,2 \mathrm{mg} / \mathrm{dl}$ para las mismas muestras con el método combinado).

\section{Estudio de estabilidad}

El estudio de la estabilidad de los calibradores combinados de Gal/Phe en sangre seca sobre papel de filtro mostró los resultados siguientes al 


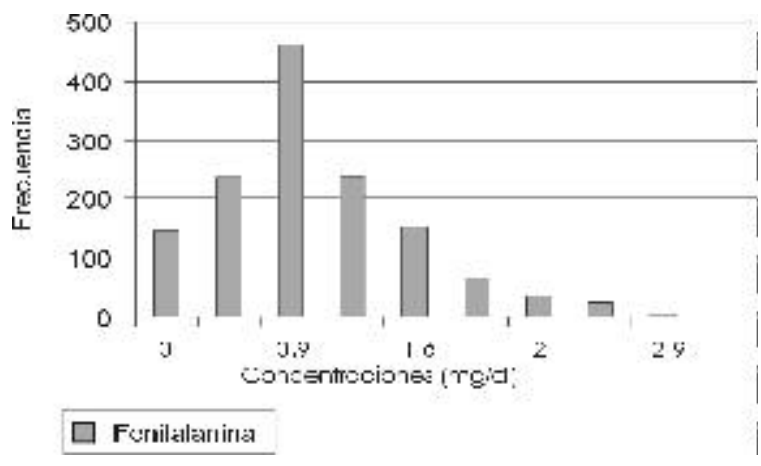

Figura 3. Distribución de frecuencias de concentración de fenilalanina en sangre seca sobre papel de filtro recolectada en recién nacidos.

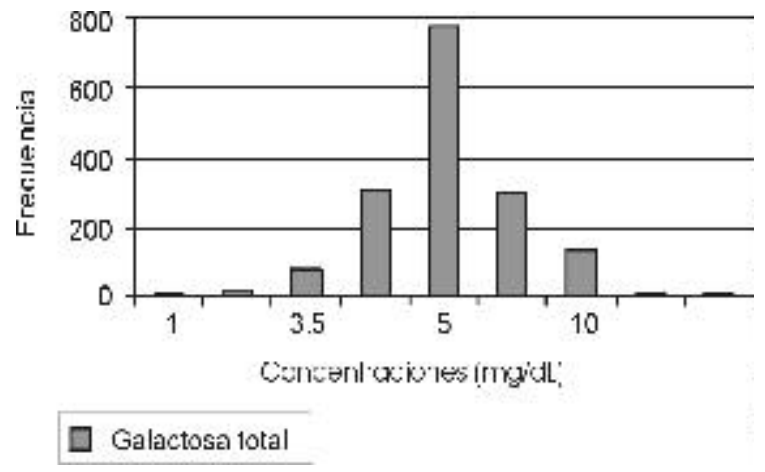

Figura 4. Distribución de galactosa total en sangre seca sobre papel de filtro recolectadas en recién nacidos.

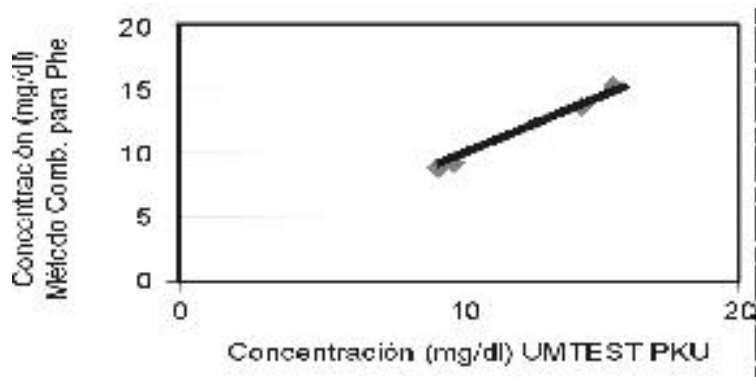

Figura 5. Análisis de correlación de cuatro muestras analizadas por el UMTEST PKU y el método combinado para fenilalanina.

ser evaluados con el método combinado para la determinación de Phe y Gal: en C3 hubo un deterioro de la galactosa, comparándola con la concentración inicial de un 10\%, a los 7 días de almacenada; a los 15 días, la pérdida fue de un $37 \%$ con respecto a la concentración esperada; en C4 se evaluó la estabilidad de la galactosa con respecto a su concentración inicial, con una pérdida de concentración de $8,7 \%$ a los 7 días de almacenada; a los 15 días, la pérdida fue de un $15 \%$. La evaluación de la galactosa se extendió hasta un período de tiempo de 15 días para C3 y 30 días para C4, pues se evidencia una pérdida real de las concentraciones de este analito. La concentración de fenilalanina no se afectó en estas condiciones (C3 y C4). El estudio de los calibradores combinados, almacenados en $\mathrm{C} 1$ y C2, se extendió a un período de 18 meses, con una adecuada estabilidad en su concentración durante este tiempo.

\section{Discusión}

Este método, que combina la cuantificación de galactosa total y fenilalanina a partir de una muestra común, permite ahorros significativos en comparación con los métodos simples. Los costos que se economizarían con este método se explican al compartir el trabajo manual en la recolección y organización de las muestras, en la perforación de los discos, la extracción de los analitos por evaluar y el uso de algunos reactivos y equipos en común.

Los ensayos microfluorimétricos continúan teniendo una amplia capacidad de aplicación en los tamizajes neonatales de enfermedades metabólicas (6). El empleo de volúmenes pequeños en este ensayo combinado no afecta la sensibilidad y la reproducibilidad del método y provee una metodología económica, confiable y, por tanto, de fácil aplicación en los laboratorios dedicados al tamizaje de estas enfermedades.

La técnica de recolectar sangre en papel de filtro facilita la realización de programas de detección precoz de diferentes enfermedades de origen genético y metabólico en niños recién nacidos, ya que no se requiere punción venosa, pues las muestras pueden obtenerse por punción del talón o digital y la cantidad de sangre necesaria es pequeña. Por otra parte, simplifica el traslado de las muestras desde lugares distantes hasta un laboratorio central, sin necesidad de condiciones especiales e implica un menor riesgo biológico (7).

El tiempo que media entre el momento de recolección de la muestra y su análisis en el 
laboratorio y las diferentes condiciones de temperatura a las cuales están sometidas las muestras durante su transporte y almacenamiento pudieran comprometer los resultados analíticos del ensayo; es por ello que consideramos útil para nuestro estudio evaluar la estabilidad de los calibradores combinados de Gal/Phe, ya que es muy importante conocer su estabilidad en el tiempo bajo diferentes condiciones de almacenamiento, con el objetivo de garantizar la confiabilidad del diagnóstico.

Estos calibradores combinados de Gal/Phe, preparados en sangre total, mostraron una adecuada estabilidad en papel de filtro y pueden ser almacenados hasta siete días a temperatura ambiente; después de este tiempo, las concentraciones de galactosa comienzan a mermar y la pérdida sería mayor de un $10 \%$, comprometiendo las características analíticas y diagnósticas del método. La estabilidad de un analito se toma comúnmente como el tiempo durante el cual ocurre un cambio de $10 \%$ de su concentración con respecto a la concentración original (7); entre $-20^{\circ} \mathrm{C}$ y $4^{\circ} \mathrm{C}$ estos calibradores combinados de GAL/Phe pueden almacenarse durante 1 año. Durante este período, no se observaron cambios mayores o iguales a $10 \%$ en su concentración.

En los ensayos de inhibición bacteriana, la interferencia por antibióticos constituye un serio problema, no así en los ensayos fluorométricos; sin embargo, éstos se ven afectados por señales de fluorescencias inespecíficas y reacciones cruzadas con los componentes de algunos medicamentos (8). Los 14 antibióticos evaluados en nuestro método no evidenciaron ser causa de interferencia.

A pesar del evidente beneficio que implica para el niño, la familia y la sociedad la disponibilidad de programas masivos de tamizaje de errores innatos del metabolismo, éstos han sido un privilegio de países con alto grado de desarrollo económico, debido, en parte, al alto costo de los reactivos necesarios para ejecutarlos; sin embargo, la organización de programas con carácter masivo en países en vías de desarrollo se ha dificultado no sólo por las condiciones económicas y las enfermedades de origen infeccioso que enfrentan las autoridades de salud de estos países, sino por la carencia de tecnologías de bajo costo y complejidad aceptable (9).

Con nuestro estudio queremos demostrar cuán efectivo resultaría utilizar el eluido obtenido de una misma muestra para varios análisis, lo cual reduce los costos implícitos en la toma de muestras.

Una vez establecido y puesto en marcha un programa masivo para la detección de la fenilcetonuria (3), en la cual la toma de muestra representa un costo significativo, este método permite incorporar al programa la detección precoz de galactosemia, sin aumentar sustancialmente los costos del programa, lo que justificaría el tamizaje neonatal de una enfermedad metabólica de baja incidencia (4).

\section{Referencias}

1. Beutler E. Galactosemia: screening and diagnosis. Clin Biochem 1991;24:293-300.

2. Hoffman GL, Laessig RH, Jhassemer D, Makowski E. Dual-channel continious-flow systems for determination of phenylalanine and galactose. Clin Chem 1984;30 /2:287-90.

3. McCaman MW, Robins E. Fluorometric method for the determination of phenylalanine in serum. $\mathrm{J} \mathrm{Lab}$ Clin Med 1962;59:885.

4. Yamaguchi A, Fukushi M, Mizushima Y, Shimizu Y, Takasugi N, Arashima S, et al. Microassay for screening newborn for galactosemia with use of fluorometric microplate reader. Clin Chem 1989;35:9.

5. Machado C, Solís RL, Bécquer D, Cazanave J, Fernández JL. The ultramicroanalytical system (SUMA) as a new approach to the newborn screening for hyperphenylalaninemias. En: Levy HL, Hermos RJ, Grady GF, editors. Proceedings of the Third Meeting of the International Society for Neonatal Screening; 1996, Oct 20-23. Boston, Massachusets: IKON/MAP; 1996. p.238-9.

6. Bickel HC, Bachman C, Beckers E. Neonatal mass screening for metabolic disorders. Eur J Pediatrics 1981; 137:133-9.

7. Levy HL, Simmons JR, McCready RA. Stability of amino acids and galactose in the newborn screening filter paper blood specimen. J Pediatrics 1985;107:75760 . 
8. Holton JB. A large scale comparison of the bacteriological inhibition assay and the automated fluorimetric method for phenylketonuria screening. Ann Clin Biochem 1972; 9:118.

9. Fernádez JL. Pesquisa neonatal en países en vías de desarrollo. En: Cornejo V, Raimann E, Colombo M, editores. Libro de resumen II Congreso Latinoamericano de Errores Innatos del Metabolismo y Pesquisa Neonatal; 1999, 24-27 Octubre. Caupolicán: Servicios Gráficos; 1999. p.29. 